\title{
Estimation of the Feedback Reliability for IB-DFE Receivers
}

\author{
Fábio Silva, ${ }^{1}$ Rui Dinis, ${ }^{1,2}$ and Paulo Montezuma ${ }^{2,3}$ \\ ${ }^{1}$ Wireless Communications Group, Instituto de Telecomunicações, Av. Rovisco Pais 1, 1049-001 Lisboa, Portugal \\ ${ }^{2}$ DEE, FCT/UNL, Monte de Caparica, 2829-516 Caparica, Portugal \\ ${ }^{3}$ Center of Technologies and Systems, UNINOVA, Campus da FCT/UNL, Monte de Caparica, 2829-516 Caparica, Portugal
}

Correspondence should be addressed to Fábio Silva, fabio.silva@lx.it.pt

Received 26 April 2011; Accepted 6 June 2011

Academic Editors: G. DeJean and C. Pomalaza-Ráez

Copyright (C) 2011 Fábio Silva et al. This is an open access article distributed under the Creative Commons Attribution License, which permits unrestricted use, distribution, and reproduction in any medium, provided the original work is properly cited.

single-carrier (SC) modulation with frequency-domain equalization (FDE) combined with iterative (turbo) FDE schemes has been shown to be suitable for cyclic-prefix (CP)-assisted block transmission within broadband wireless systems. Its excellent performance in severely time-dispersive channels, makes it a promising candidate for future broadband wireless systems. In this paper we investigated the impact of the correlation factor estimation in the performance of iterative block decision feedback equalization (IB-DFE) receivers. Since the correlation factor represents a key parameter to ensure the good performance of these receivers, reliable estimates of this factor are needed in the feedback loop. We present several methods to estimate the correlation coefficient. We also propose a technique to compensate the inaccuracy of the estimation.

\section{Introduction}

Future wireless systems are required to support high quality of service at high data rates, for which we can have severely time-dispersion effects associated to the multipath propagation. It is known that block transmission techniques, with appropriate cyclic extensions and employing FDE techniques, are excellent candidates for severely time-dispersive channels $[1,2]$. The most popular techniques based on this concept are orthogonal frequency division multiplexing (OFDM) and single-carrier with frequency-domain equalization (SC-FDE). However, the OFDM signals have high envelope fluctuations and a high peak-to-mean envelope power ratio (PMEPR) leading to amplification difficulties. An alternative to OFDM modulations are SC modulations using FDE and CP-assisted block transmission techniques, especially for the uplink of broadband wireless systems $[1,2]$. The performances and overall implementation complexities are similar to SC-FDE and OFDM $[1,2]$.

Typically the receiver for SC-FDE schemes is a linear FDE. However, it is known that nonlinear equalizers outperform linear equalizers [3]. iterative block decision feedback equalizer (IB-DFE) [4] is a promising iterative
FDE technique for SC-FDE that was first proposed in [5] and extended to diversity scenarios [6] and layered spacetime schemes [7]. IB-DFE receivers can be regarded as iterative DFE receivers with the feedforward and the feedback operations implemented in the frequency domain. Since the feedback loop takes into account not just the hard decisions for each block, but also the overall block reliability we have small error propagation. Consequently, the IBDFE techniques offer much better performances than the noniterative methods, with performances that can be close to the matched filter bound (MFB) [8] and can be regarded as low complexity turbo equalization schemes $[9,10]$, since the feedback loop uses the equalizer outputs instead of the channel decoder outputs. True turbo FDE schemes can also be designed based on the IB-DFE concept $[11,12]$.

In this paper, we study the impact of the correlation coefficient estimation on the asymptotic performance of IBDFE schemes. Several methods to estimate the correlation coefficient are derived as well as a proposed technique to compensate the inaccuracy of the estimations.

This paper is organized as follows: conventional and turbo IB-DFE receivers are described in Section 2. Various methods to estimate the correlation coefficient are presented 
in Section 3. The compensation technique is also included. A set of performance results is presented in Section 4, and Section 5 is concerned with the conclusion of this paper.

\section{System Description}

2.1. Transmitted and Received Signals. We consider an SCFDE modulation where the data is transmitted in blocks of $N$-useful modulation symbols $\left\{s_{n} ; n=0, \ldots, N-1\right\}$, resulting from a direct mapping of the original data bits into a selected signal constellation, for example, QPSK. A cyclic prefix with length longer than the channel impulse response is posteriorly appended, resulting the transmitted signal $\left\{s_{n} ; n=-N_{G}, \ldots, N-1\right\}$, where $N_{G}$ denotes the number of guard samples. This signal is transmitted over a time-dispersive channel. The received signal is sampled at the receiver where the $\mathrm{CP}$ samples are removed, leading in the time domain the samples $\left\{y_{n} ; n=0, \ldots, N-1\right\}$. The corresponding frequency-domain block, obtained after an appropriate size- $N$ discrete fourier transform (DFT) operation, is $\left\{Y_{k} ; k=0,1, \ldots, N-1\right\}$, where $Y_{k}$ can be written as

$$
Y_{k}=S_{k} H_{k}+N_{k}
$$

where $H_{k}$ denotes the overall channel frequency response for the $k$ th frequency of the $m$ th time block, and $N_{k}$ represents channel noise term in frequency-domain.

2.2. IB-DFE Basic Receiver Structure. For the sake of simplicity, we will assume in this section perfect synchronization conditions.

In SC-FDE to deal with the channel effects, we can employ a linear FDE. However, the performance can be improved if the linear FDE is replaced with an IB-DFE [5], whose basic structure is depicted in Figure 1. In this case, for the $i$ th iteration, the frequency-domain block at the output of the equalizer is $\left\{\widetilde{S}_{k}^{(i)} ; k=0,1, \ldots, N-1\right\}$, with

$$
\widetilde{S}_{k}^{(i)}=F_{k}^{(i)} Y_{k}-B_{k}^{(i)} \widehat{S}_{k}^{(i-1)}
$$

where $\left\{F_{k}^{(i)} ; k=0,1, \ldots, N-1\right\}$ are the feedforward coefficients, and $\left\{B_{k}^{(i)} ; k=0,1, \ldots, N-1\right\}$ are the feedback coefficients. $\left\{\hat{S}_{k}^{(i-1)} ; k=0,1, \ldots, N-1\right\}$ is the DFT of the block $\left\{\hat{s}_{n}^{(i-1)} ; n=0,1, \ldots, N-1\right\}$, with $\hat{s}_{n}$ denoting the "hard decision" of $s_{n}$ from the previous FDE iteration. It can be shown that the optimum coefficients $B_{k}$ and $F_{k}$ that maximize the overall SNR (instantaneous signal-to-noise ratio) associated to the samples $\widetilde{S}_{k}$ are [6]

$$
\begin{gathered}
B_{k}^{(i)}=\rho\left(F_{k}^{(i)} H_{k}-1\right), \\
F_{k}^{(i)}=\frac{\kappa H_{k}^{*}}{\alpha+\left(1-\left(\rho_{m}^{(i-1)}\right)^{2}\right)\left|H_{k}\right|^{2}} ;
\end{gathered}
$$

respectively, where $\alpha=E\left[\left|N_{k}\right|^{2}\right] / E\left[\left|S_{k}\right|^{2}\right]$ (common to all data blocks), and $\kappa$ selected to guarantee that $1 / N \sum_{k=0}^{N-1} F_{k}^{(i)} H_{k}=1$.

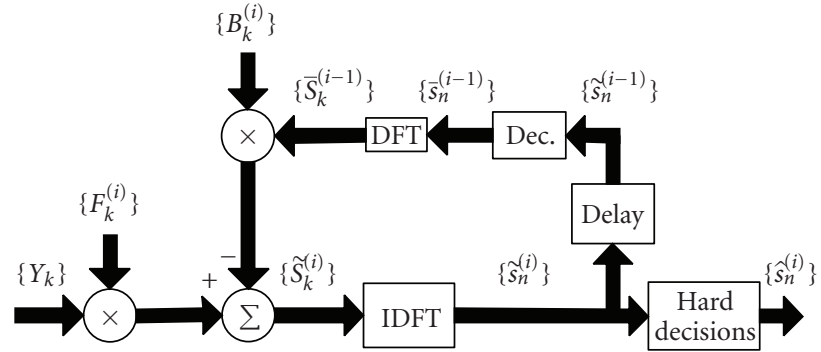

FIGURE 1: Basic receiver structure of an IB-DFE.

As we can see from (3) and (4), the correlation factor $\rho^{(i-1)}$ is a key parameter for the good performance of IB-DFE receivers, since it supplies a blockwise reliability measure of the estimates, associated to the previous iteration, that are employed in the feedback loop. This is done in the feedback loop by taking into account the "hard-decisions" for each block plus the overall block reliability, which reduces error propagation problems. The correlation factor $\rho^{(i-1)}$ is defined as

$$
\rho_{m}^{(i-1)}=\frac{E\left[\hat{\boldsymbol{s}}_{n}^{(i-1)} s_{n}^{*}\right]}{E\left[\left|s_{n}\right|^{2}\right]}=\frac{E\left[\hat{S}_{k}^{(i-1)} S_{k}^{*}\right]}{E\left[\left|S_{k}\right|^{2}\right]}
$$

where the block $\left\{\hat{\boldsymbol{s}}_{n}^{(i-1)} ; n=0,1, \ldots, N-1\right\}$ denotes the data estimates associated to the previous iteration, that is, the hard decisions associated to the time-domain block at the output of the FDE, $\left\{\widetilde{s}_{n}^{(i)} ; n=0,1, \ldots, N-1\right\}=$ IDFT $\left\{\tilde{S}_{k}^{(i)} ; k=0,1, \ldots, N-1\right\}$.

It should be noted that (2) can be written as

$$
\tilde{S}_{k}^{(i)}=F_{k}^{(i)} Y_{k}-B_{k}^{\prime(i)} \bar{S}_{k, \text { Block }}^{(i-1)}
$$

where $B_{k}^{(i)}=B_{k}^{(i)} / \rho_{m}^{(i-1)}$ and $\bar{S}_{k, \text { Block }}^{(i-1)}=\rho_{m}^{(i-1)} \hat{S}_{k}^{(i-1)}$ (as stated before, $\rho^{(i-1)}$ can be considered as the blockwise reliability of the estimates $\left.\left\{\widehat{S}_{k}^{(i-1)} ; k=0,1, \ldots, N-1\right\}\right)$.

To improve the IB-DFE performance, it is possible to use "soft decisions," $\bar{s}_{n}^{(i)}$, instead of "hard decisions," $\hat{s}_{n}^{(i)}$. Under these conditions, the "blockwise average" is substituted by "symbol averages" [11]. This can be done by using $\left\{\bar{S}_{k, \text { Symbol }}^{(i-1)} ; k=0,1, \ldots, N-1\right\}$ instead of $\left\{\bar{S}_{k, \text { Block }}^{(i-1)} ; k=\right.$ $0,1, \ldots, N-1\}=\operatorname{DFT}\left\{\bar{s}_{n, \text { Symbol }}^{(i-1)} ; n=0,1, \ldots, N-1\right\}$, where $\bar{s}_{n, \text { Symbol }}^{(i-1)}$ denotes the average symbol values conditioned to the FDE output from previous iteration, $\widetilde{s}_{n}^{(i-1)}$. To simplify the notation, we will replace $\bar{s}_{n, \text { Symbol }}^{(i-1)}$ with $\bar{s}_{n}^{(i-1)}$ in the following equations.

For QPSK constellations, the conditional expectations associated to the data symbols for the $i$ th iteration are given by

$$
\bar{s}_{n}^{(i)}=\tanh \left(\frac{L_{n}^{I(i)}}{2}\right)+j \tanh \left(\frac{L_{n}^{Q(i)}}{2}\right)=\rho_{n}^{I} \widehat{s}_{n}^{I}+j \rho_{n}^{Q} \widehat{s}_{n}^{Q},
$$


with the loglikelihood ratios (LLRs) of the "in-phase bit" and the "quadrature bit," associated to $s_{n}^{I}$ and $s_{n}^{Q}$, given by

$$
\begin{gathered}
L_{n}^{I(i)}=\frac{2}{\sigma_{i}^{2}} \widetilde{s}_{n}^{I(i)}, \\
L_{n}^{Q(i)}=\frac{2}{\sigma_{i}^{2}} \widetilde{s}_{n}^{Q(i)},
\end{gathered}
$$

respectively, with

$$
\sigma_{i}^{2}=\frac{1}{2} E\left[\left|s_{n}-\widetilde{s}_{n}^{(i)}\right|^{2}\right] \approx \frac{1}{2 N} \sum_{n=0}^{N-1}\left|\widehat{s}_{n}^{(i)}-\widetilde{s}_{n}^{(i)}\right|^{2}
$$

with the signs of $L_{n}^{I}$ and $L_{n}^{Q}$ defining the hard decisions $\hat{s}_{n}^{I}=$ \pm 1 and $\hat{s}_{n}^{Q}= \pm 1$, respectively. In (7), $\rho_{n}^{I}$ and $\rho_{n}^{Q}$ denote the reliabilities related to the "in-phase bit" and the "quadrature bit" of the $n$th symbol and are given by

$$
\begin{gathered}
\rho_{n}^{I(i)}=\frac{E\left[s_{n}^{I} \hat{s}_{n}^{I}\right]}{E\left[\left|s_{n}^{I}\right|^{2}\right]}=\left|\tanh \left(\frac{L_{n}^{I(i)}}{2}\right)\right|, \\
\rho_{n}^{Q(i)}=\frac{E\left[s_{n}^{Q} \hat{s}_{n}^{Q}\right]}{E\left[\left|s_{n}^{Q}\right|^{2}\right]}=\left|\tanh \left(\frac{L_{n}^{Q(i)}}{2}\right)\right| .
\end{gathered}
$$

Therefore, the correlation coefficient employed in the feedforward coefficients will be given by

$$
\rho^{(i)}=\frac{1}{2 N} \sum_{n=0}^{N-1}\left(\rho_{n}^{I(i)}+\rho_{n}^{Q(i)}\right) .
$$

Obviously, for the first iteration $\rho_{n}^{I(0)}=\rho_{n}^{Q(0)}=0$, and consequently $\bar{s}_{n}=0$.

We may note that the receiver that employs "blockwise reliabilities" is referred as IB-DFE with "hard decisions," while the receiver that employs "symbol reliabilities" is referred as IB-DFE with "soft decisions." The feedforward coefficients used in both types of IB-DFE receivers are still given by (4), but the feedback loop of the IB-DFE with "hard decisions" uses the estimated data block, weighted by a reliability coefficient common to the entire block, while for IB-DFE with "soft decisions," the feedback loop uses a different reliability coefficient for each symbol (changes from bit to bit).

With a conventional IB-DFE receiver, the log-likelihood values are computed on a symbol-by-symbol basis (i.e., we do not need to perform the channel decoding in the feedback loop). As an alternative, we can define a turbo IB-DFE that employs the channel decoder outputs instead of the uncoded "soft decisions" in the feedback loop. The main difference between conventional IB-DFE and turbo IB-DFE is in the decision device; in the first case, the decision device is a symbol-by-symbol soft decision (for QPSK constellation this corresponds to the hyperbolic tangent, as in (7)); for the turbo IB-DFE, a soft-in, soft-out (SISO) channel decoder is employed in the feedback loop. The SISO block can be implemented as defined in [13] and provides the LLRs of both the "information bits" and the "coded bits." The input of the SISO block is LLRs of the "coded bits" at the FDE output, given by $L_{n}^{I(i)}$ and $L_{n}^{Q(i)}$.

\section{Correlation Coefficient Estimation}

As shown in the previous section, the correlation factor is a key parameter for the good performance of IB-DFE receivers, since it supplies a blockwise reliability measure of the estimates employed in the feedback loop. However, contrarily to the channel frequency response, it changes from block to block and iteration to iteration. Therefore, it cannot be computed using reference blocks and needs to be obtained from the equalized output.

In this section, we present several methods to estimate the correlation coefficient. The correlation coefficient $\rho^{(i)}$ was defined in (5), and for the sake of simplicity, the iteration number $i$ will be ignored in the following equations. Therefore, (5) can be rewritten as

$$
\rho=\frac{E\left[\hat{s}_{n} s_{n}^{*}\right]}{E\left[\left|s_{n}\right|^{2}\right]}=\frac{E\left[\hat{S}_{k} S_{k}^{*}\right]}{E\left[\left|S_{k}\right|^{2}\right]} .
$$

Let us consider the transmitted symbols $\left\{s_{n} ; n=0, \ldots, N-\right.$ 1 ) corresponding to a QPSK constellation, under a Gray mapping rule. Consequently, the $s_{n}$ may be written as

$$
s_{n}=s_{n}^{I}+s_{n}^{Q}= \pm d \pm j d,
$$

in which $s_{n}^{I}=\operatorname{Re}\left\{s_{n}\right\}$ is the "in-phase" component of $s_{n}$, and $s_{n}^{Q}=\operatorname{Im}\left\{s_{n}\right\}$ is the "quadrature" component of $s_{n}$. Also, $d$ is given by $d=D / 2$, where $D$ denotes the minimum Euclidean distance between two constellation symbols. Under these conditions, $E\left[\left|s_{n}\right|^{2}\right]=D^{2} / 4$. The time-domain samples at the FDE's output are given by

$$
\widetilde{s}_{n}=\widetilde{s}_{n}^{I}+\widetilde{s}_{n}^{Q}=s_{n}+\theta_{n},
$$

where $\theta_{n}$ denotes the global error term, which is Gaussiandistributed, with zeromean. The symbol estimates are then given by

$$
\widehat{s}_{n}=s_{n}+\vartheta_{n}^{I}+j \vartheta_{n}^{Q},
$$

where $\vartheta_{n}^{I}$ denotes the error coefficient in $\hat{s}_{n}^{I}$, and $\vartheta_{n}^{Q}$ denotes the error coefficient in $\hat{s}_{n}^{Q}$. Clearly, if $\hat{s}_{n}^{I}$ and $\hat{s}_{n}^{Q}$ have no errors, then $\vartheta_{n}^{I}$ and $\vartheta_{n}^{Q}$ are null. On the other hand, if there are errors, then the value of $\vartheta_{n}^{I}$ and/or $\vartheta_{n}^{Q}$ will be $\pm D$. Consequently, $\vartheta_{n}^{I}$ and $\vartheta_{n}^{Q}$ are random variables, with values $0,+D$ and $-D$ with probabilities $1-2 P_{e}, P_{e}$ and $P_{e}$ respectively, where $P_{e}$ denotes the bit error rate. Therefore, it can be shown that

$$
\rho=1-2 P_{e} .
$$

Naturally, in practice, we do not know the transmitted symbols $\left\{s_{n} ; n=0, \ldots, N-1\right\}$.

3.1. Method I: Estimation Based on the BER Estimate. The total variance of the overall noise plus residual ISI, $\sigma_{E q}^{2}$, is given by

$$
\sigma_{E q}^{2}=\frac{1}{2} E\left[\left|s_{n}-\tilde{s}_{n}\right|^{2}\right]
$$


The exact value of $\sigma_{E q}^{2}$ can not be used in practice, because we do not know the transmitted symbols $\left\{s_{n} ; n=0, \ldots, N-1\right\}$. Therefore, we may use an approximated value of $\sigma_{E q}^{2}$, given by

$$
\widehat{\sigma}_{E q}^{2}=\frac{1}{2 N} \sum_{n=0}^{N-1}\left|\widehat{s}_{n}-\widetilde{s}_{n}\right|^{2} .
$$

Provided that the numbers of decision errors in $\widehat{s}_{n}$ are small and $N$ is high, we have

$$
\sigma_{E q}^{2} \approx \hat{\sigma}_{E q}^{2} .
$$

For a QPSK constellation, the estimated BER, denoted by $\hat{P}_{e}$, can be approximated by

$$
\hat{P}_{e} \approx Q\left(\frac{1}{\hat{\sigma}_{E q}}\right),
$$

and, from (17), the estimated value of $\rho$ will be

$$
\hat{\rho}=1-2 \hat{P}_{e} .
$$

3.2. Method II: Estimation Based on the LLR. This technique consists in the estimation of the correlation coefficient with resort to the log-likelihood ratio (LLRs), as derived in Section 2.2. However, as in method I, an approximated value for the variance of channel and interference noise is considered, instead of the optimum value

$$
\hat{\sigma}_{E q}^{2}=\frac{1}{2 N} \sum_{n=0}^{N-1}\left|\widehat{s}_{n}-\widetilde{s}_{n}\right|^{2} .
$$

The calculation of the LLRs of the "in-phase bit" and the "quadrature bit", associated to $s_{n}^{I}$ and $s_{n}^{Q}$, are based in (8) and (9), respectively, but with $\widehat{\sigma}_{E q}^{2}$ in place of $\sigma_{E q}^{2}$.

Consequently, the reliabilities related to the "in-phase bit" and the "quadrature bit" of the $n$th symbol, are based on (11), respectively, but with $\widehat{L}_{n}^{I}$ and $\widehat{L}_{n}^{Q}$ in place of $L_{n}^{I}$ and $L_{n}^{Q}$.

Therefore, the estimated value of the correlation coefficient will be given by

$$
\hat{\rho}=\frac{1}{2 N} \sum_{n=0}^{N-1}\left(\hat{\rho}_{n}^{I}+\hat{\rho}_{n}^{Q}\right) .
$$

3.3. Method III: Estimation Based on the MSE. This estimation method is based on the same technique employed in method I, given by (22). However, unlike method I, the BER calculation employs the mean-squared error (MSE) of the equalized samples, instead of an approximated value for the variance of the channel and interference noise. Therefore, in method III, the estimated bit error rate denoted by $\hat{P}_{e}$ is given by

$$
\hat{P}_{e}=Q\left(\sqrt{\frac{|\gamma|^{2} \cdot E\left|S_{k}\right|^{2}}{\sigma_{\mathrm{MSE}}^{2}}}\right) .
$$

Once again, assuming a QPSK constellation with a gray mapping and $s_{n}= \pm 1 \pm j$, then (25) can be written as

$$
\hat{P}_{e}=Q\left(\frac{|\gamma|}{\sqrt{\sigma_{\mathrm{MSE}}^{2}}}\right),
$$

where $\sigma_{\mathrm{MSE}}^{2}$ corresponds to the MSE and is defined as

$$
\sigma_{\mathrm{MSE}}^{2}=\frac{1}{N^{2}} \sum_{k=0}^{N-1} \Theta_{k},
$$

with

$$
\Theta_{k}=E\left[\left|\tilde{S}_{k}-S_{k}\right|^{2}\right] .
$$

By combining (1) and (6), and assuming $E\left[\left|N_{k} \cdot \Delta_{k}^{*}\right|\right]=0$, $E\left[\left|N_{k} \cdot S_{k}^{*}\right|\right]=0$, and $E\left[\left|S_{k} \cdot \Delta_{k}^{*}\right|\right]=0$, we can rewrite (28) as

$$
\begin{aligned}
\Theta_{k}= & E\left[\left|\tilde{S}_{k}-S_{k}\right|^{2}\right] \\
= & E\left[\left|F_{k} S_{k} H_{k}+F_{k} N_{k}-B_{k} \rho \hat{S}_{k}-S_{k}\right|^{2}\right] \\
= & E\left[\left|F_{k} H_{k}-B_{k} \rho^{2}-1\right|^{2}\right] \cdot 2 \cdot \sigma_{S}^{2}+E\left[\left|F_{k}\right|^{2}\right] \cdot 2 \cdot \sigma_{N}^{2} \\
& +E\left[\left|B_{k}\right|^{2}\right] \rho^{2} \cdot\left(1-\rho^{2}\right) \cdot 2 \cdot \sigma_{S}^{2},
\end{aligned}
$$

where the variance of the transmitted frequency-domain data symbols is given by

$$
\sigma_{S}^{2}=\frac{E\left[\left|S_{k}\right|^{2}\right]}{2},
$$

the variance of the channel noise is obtained by

$$
\sigma_{N}^{2}=\frac{E\left[\left|N_{k}\right|^{2}\right]}{2},
$$

the expected value of the error term for the $k$ th frequencydomain "hard decision" estimate is defined as

$$
E\left[\left|\Delta_{k}\right|^{2}\right]=\sigma_{S}^{2}\left(1-\rho^{2}\right),
$$

and the estimated value of $\rho$ can be expressed by

$$
\hat{\rho}=1-2 \widehat{P}_{e},
$$

with $\hat{P}_{e}$ given by (26).

\section{Performance Results}

In the following, we present a set of results obtained during the research of the impact of the correlation coefficient estimation on the performance of IB-DFE receivers, for 10 iterations, for a given channel realization. We consider uncoded transmissions of FFT blocks with $N=1024$ data symbols, selected from a QPSK constellation under a gray mapping rule. Similar results were observed for other values of $N$, provided that $N \gg 1$. 


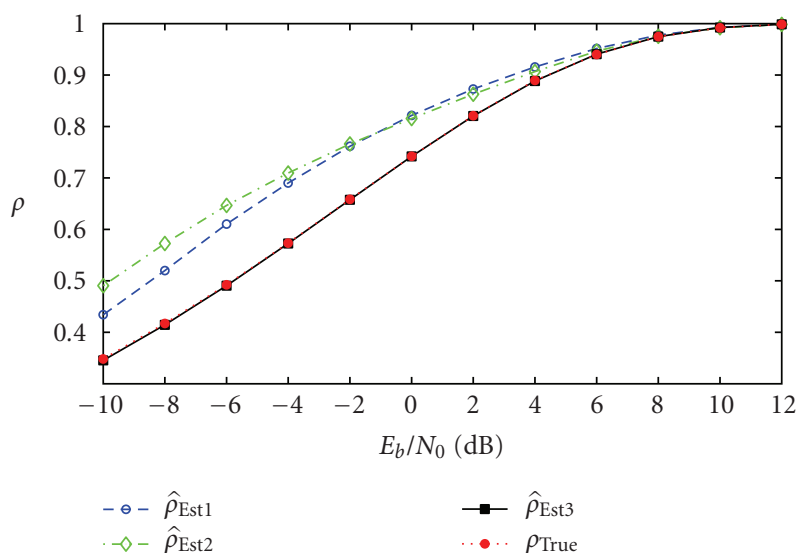

FIGURE 2: Evolution of $\rho$ as function of the $E_{b} / N_{0}$ for methods I, II, and III.

\subsection{Results without Correlation Coefficient Compensation.} Figure 2 presents the evolution of the correlation coefficient for values of $E_{b} / N_{0}$. The results show the differences between the ideal value of the correlation coefficient (obtained with (13)), and the estimated value for methods I, II, and III (obtained by (22), (24) and (33), resp.). As we can see, for methods I and II, the $\hat{\rho}$ curve suffered a deviation from the curve corresponding to the evolution of the optimum (true) correlation coefficient. This is caused by the use of the approximated value $\hat{\sigma}_{E q}$ in the correlation coefficient calculation of both methods, which leads to an optimistic $P_{e}$ derivation for method I, while as for method II results in optimistic versions of the LLRs. Consequently, these effects will originate optimistic estimates of the transmitted symbols in the feedback loop. In the case of method III, the $\hat{\rho}$ curve is very close to the optimum (true) $\rho$ curve. This means that the reliability measure estimation, with resort to the MSE, used in the feedback loop is very accurate. It is important to note that the curve related with method III is presented for comparison purposes only, since the variance of the channel and interference noise estimation, $\widehat{\sigma}_{E q}$, is not considered in the estimation process.

Next we present the BER performance results. Since that the results were similar for all methods, as an example, we present in Figure 3 the results related with method I. These results are expressed as function of $E_{b} / N_{0}$, where $N_{0}$ is the one-sided power spectral density of the noise, and $E_{b}$ is the energy of the transmitted bits (i.e., the degradation due to the useless power spent on the cyclic prefix is not included). These results also refer to a scenario where the IB-DFE suffers from error propagation.

Figure 4 presents the required BER to achieve $E_{b} / N_{0}=6$ $(\mathrm{dB})$ as a function of the iterations number. Clearly, there is a performance degradation after a few iterations. Although the approximation in (20) may seem reliable, the true value of $\sigma_{E q}^{2}$ is in fact higher than the estimated one due to decision errors. Consequently, the estimated BER, $\hat{P}_{e}$, is lower than the true bit error rate, $P_{e}$, leading to $\hat{\rho} \geq \rho$, that is, we are assuming that the estimates used in the feedback loop are more reliable than what they are in fact. It should be pointed

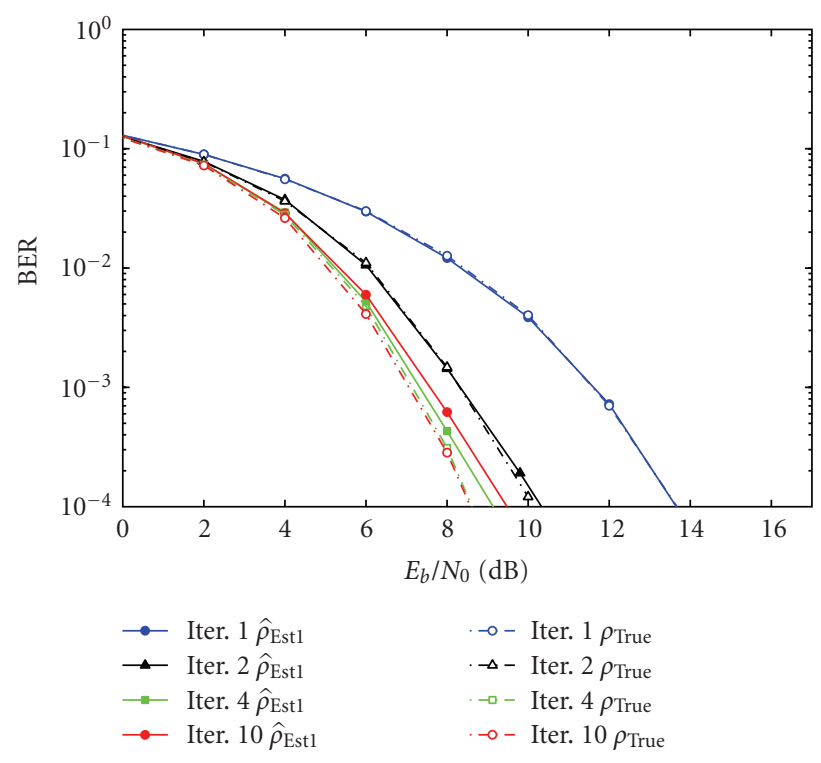

FIGURE 3: BER performance for method I.

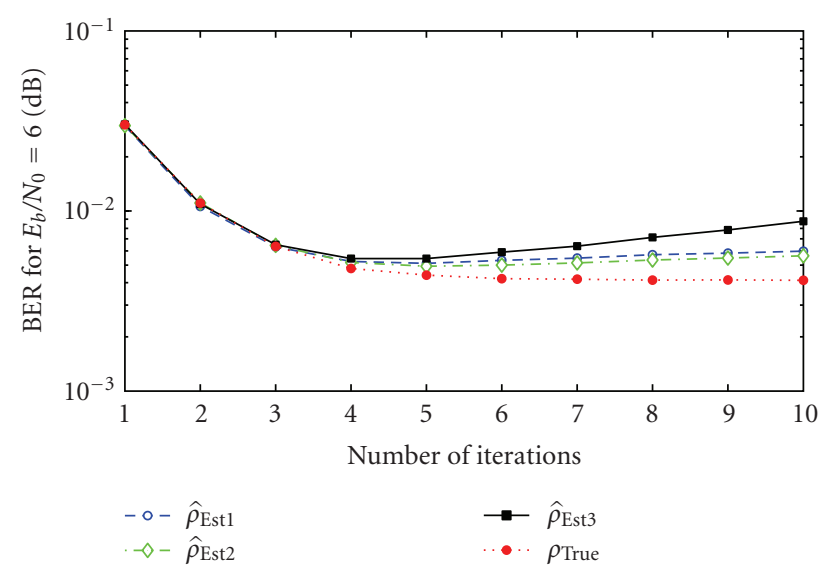

FIgURE 4: Required BER to achieve $E_{b} / N_{0}=6(\mathrm{~dB})$ as a function of the iterations number for methods I, II, and III without compensation.

out that while underestimating $\rho$ (i.e., using $\hat{\rho}<\rho$ ) leads to a slower convergence of the IB-DFE, overestimating $\rho$ (i.e., using $\hat{\rho}>\rho$ ) leads to a fast convergence but worse BER values.

\subsection{Results with Correlation Coefficient Compensation}

4.2.1. Technique Description. To overcome the problem of using an optimistic version of transmitted symbols estimates in the feedback loop, we propose a technique to compensate the inaccuracy of the correlation coefficient estimation.

The compensation factor denoted, by $\chi(\hat{\rho})$, can be expressed as $\chi(\hat{\rho})=\hat{\rho} / \rho$, where $\hat{\rho}$ is the estimated correlation coefficient derived from a given estimation method. The curves in Figure 5, obtained by simulation, show the relation between the correlation coefficient estimation, $\hat{\rho}$, and the compensation factor, $\chi(\hat{\rho})$, for methods I, II, and III. 


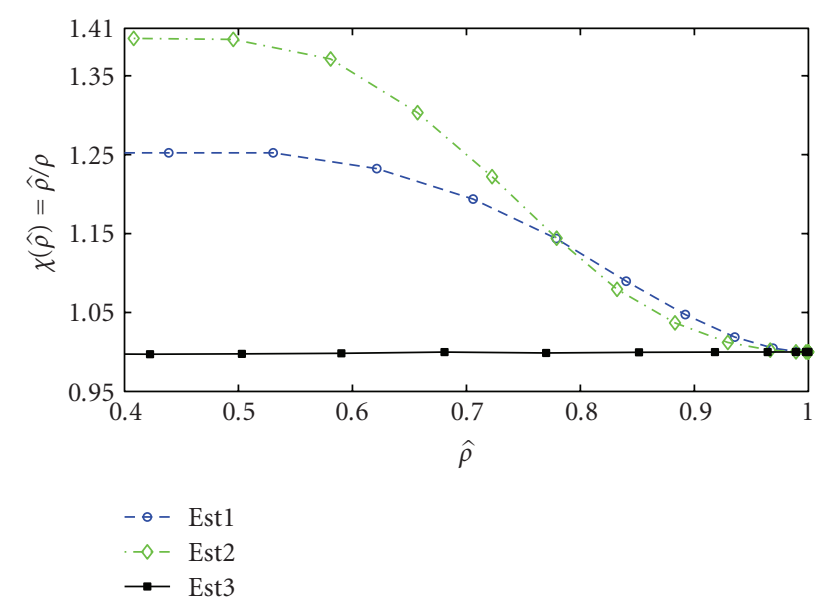

FIGURE 5: Relation between the correlation coefficient estimation and the compensation factor for methods I, II, and III.

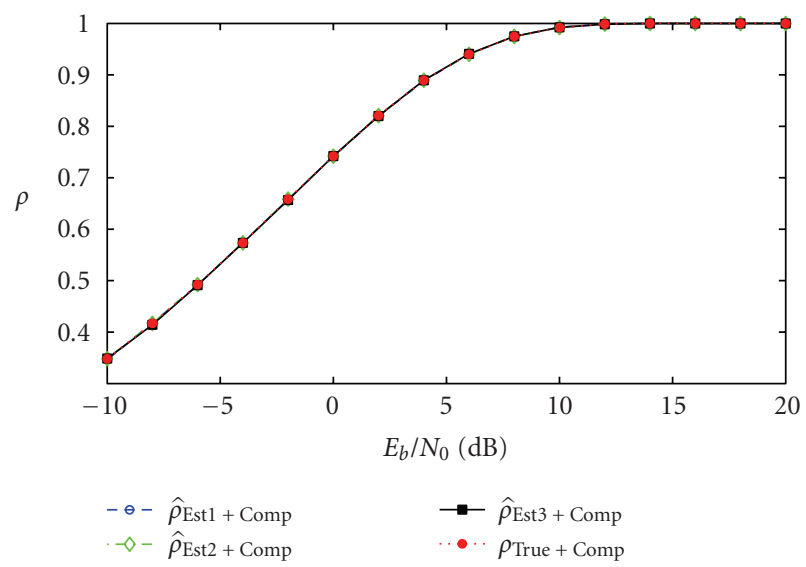

FIgURE 6: Evolution of $\rho$ as function of the $E_{b} / N_{0}$ for methods I, II, and III with compensation.

Clearly for each method, we can determine the value of the compensation factor $\chi(\hat{\rho})$ for each correspondent value of $\hat{\rho}$, by a simple interpretation of the plot presented in Figure 5 . Thus, by knowing $\chi(\hat{\rho})$, we can determine a very precise estimated version of the optimum correlation factor given by

$$
\rho \approx \rho_{\text {Est }+ \text { Comp }}=\frac{\hat{\rho}}{\chi(\hat{\rho})},
$$

where $\rho_{\text {Est }+ \text { Comp }}$ denotes the compensated correlation coefficient. Therefore, $\rho_{\text {Est+Comp }}$ can then be used in the derivation of the feedback and feedforward coefficients parameters of the IB-DFE receiver.

4.2.2. Results. We now present the performance results obtained regarding the correlation coefficient compensation. Figure 6 presents the evolution of the correlation coefficient as function of $E_{b} / N_{0}$. As we can see, for methods I and II the curves are now very close to the curve corresponding to the optimum (true) correlation coefficient evolution, when compared with the results obtained without compensation given by Figure 2. This demonstrates that the correlation

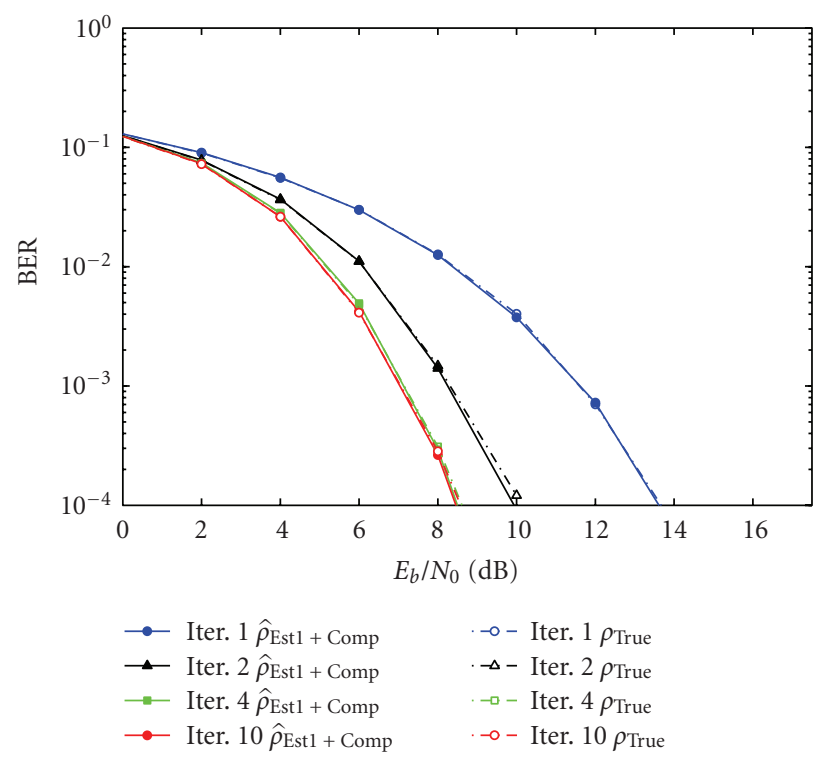

FIGURE 7: BER performance for method I with compensation.

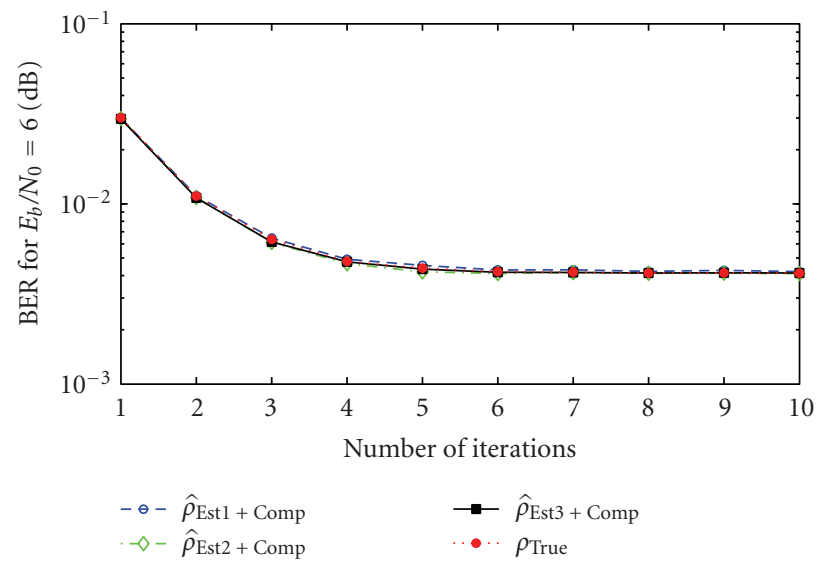

FIgURE 8: Required BER to achieve $E_{b} / N_{0}=6(\mathrm{~dB})$ as a function of the iterations number for methods I, II, and III with compensation.

coefficient inaccuracy, due to the use of optimistic estimates of the transmitted symbols in the feedback loop, can be efficiently avoided with resort to the proposed compensation technique. As expected for method III, the impact of the correlation coefficient with compensation in the evolution of the correlation coefficient is not so significative. This can be explained by the fact that correlation coefficient $\hat{\rho}$ obtained under method III was already close to the curve corresponding to the optimum (true) correlation coefficient evolution.

In Figure 7, we present the BER performance results. Once again, since that the results were similar to all methods, as an example, we present in Figure 3 the results related with method I. The improvements obtained with compensation are very significative, since that the performance curves obtained with the compensated correlation coefficient (denoted as " $\rho_{\text {Est1+Comp }}$ ", " $\rho_{\text {Est } 2+\text { Comp }}$ " and " $\rho_{\text {Est } 3+\text { Comp }}$ " in the figures) are now very close to those obtained with 
optimum (true) correlation coefficient (denoted as " $\rho$ True" in the figures), as the error propagation problem was corrected.

Figure 8 presents the required BER to achieve $E_{b} / N_{0}=6$ (dB) as a function of the iterations number. Clearly, there is a performance enhancement when compared with the correlation coefficient estimates without compensation for all methods.

\section{Conclusions}

In this paper, we studied the impact of the correlation factor estimation in the performance of IB-DFE receivers. Several methods to estimate the correlation coefficient were presented as well as a technique to compensate the estimation errors. It was shown that the inaccuracy due to the use of optimistic estimates of the transmitted symbols in the feedback loop can be efficiently avoided with resort to the proposed compensation technique.

\section{Acknowledgment}

This work was partially supported by Fundação para a Ciência e Tecnologia (pluriannual funding and ADCOD projects).

\section{References}

[1] A. Gusmão, R. Dinis, J. Conceição, and N. Esteves, "Comparison of two modulation choices for broadband wireless communications," in Proceedings of the IEEE 51st Vehicular Technology Conference (VTC '00), pp. 1300-1305, Spring, Tokyo, Japan, 2000.

[2] D. Falconer, S. Ariyavisitakul, A. Benyamin-Seeyar, and B. Eidson, "Frequency domain equalization for single-carrier broadband wireless systems," IEEE Communications Magazine, vol. 4, no. 4, pp. 1300-1305, 2000.

[3] J. Proakis, Digital Communications, McGraw-Hill, Rosehill, Calif, USA, 1995.

[4] N. Benvenuto, R. Dinis, D. Falconer, and S. Tomasin, "Single carrier modulation with non linear frequency domain equalization: an idea whose time has come-again," Proceedings of IEEE, vol. 98, no. 1, pp. 69-96, 2010.

[5] N. Benvenuto and S. Tomasin, "Block iterative DFE for single carrier modulation," Electronics Letters, vol. 39, no. 19, pp. 1300-1305, 2002.

[6] R. Dinis, A. Gusmão, and N. Esteves, "On broadband block transmission over strongly frequency-selective fading channels," in Proceedings of the IEEE Wireless, Calgary, Canada, 2003.

[7] R. Dinis, R. Kalbasi, D. Falconer, and A. H. Banihashemi, "Iterative layered space-time receivers for single-carrier transmission over severe time-dispersive channels," IEEE Communications Letters, vol. 8, no. 9, pp. 579-581, 2004.

[8] F. Coelho, R. Dinis, and P. Montezuma, "On the impact of multipath propagation and diversity in performance of iterative block decision feedback equalizers," in Proceedings of the IEEE 6th International Conference on Wireless and Mobile Computing, Networking and Communications (WiMob '10), Niagara Falls, Canada, October 2010.

[9] M. Tüchler and J. Hagenauer, "Turbo equalization using frequency domain equalizers," in Proceedings of the Allerton Conference, October 2000.
[10] M. Tüchler and J. Hagenauer, "Linear time and frequency domain turbo equalization," in Proceedings of the IEEE 54th Vehicular Technology Conference (VTC FALL '01), pp. 27732777, Atlantic City, NJ, USA, October 2001.

[11] N. Benvenuto and S. Tomasin, "Iterative design and detection of a DFE in the frequency domain," IEEE Transactions on Communications, vol. 53, no. 11, pp. 1867-1875, 2005.

[12] A. Gusmão, P. Torres, R. Dinis, and N. Esteves, "A turbo FDE technique for reduced-CP SC-based block transmission systems," IEEE Transactions on Communications, vol. 55, no. 1, pp. 16-20, 2007.

[13] B. Vucetic and J. Yuan, Turbo Codes: Principles and Applications, Kluwer Academic, Boston, Mass, USA, 2002. 

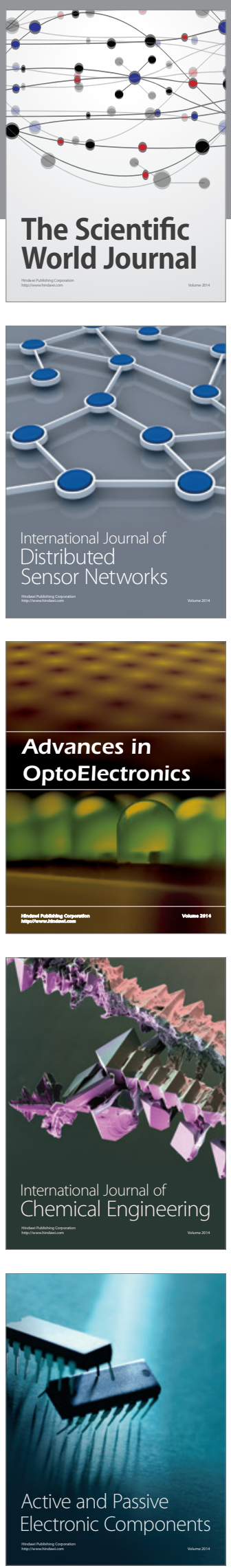
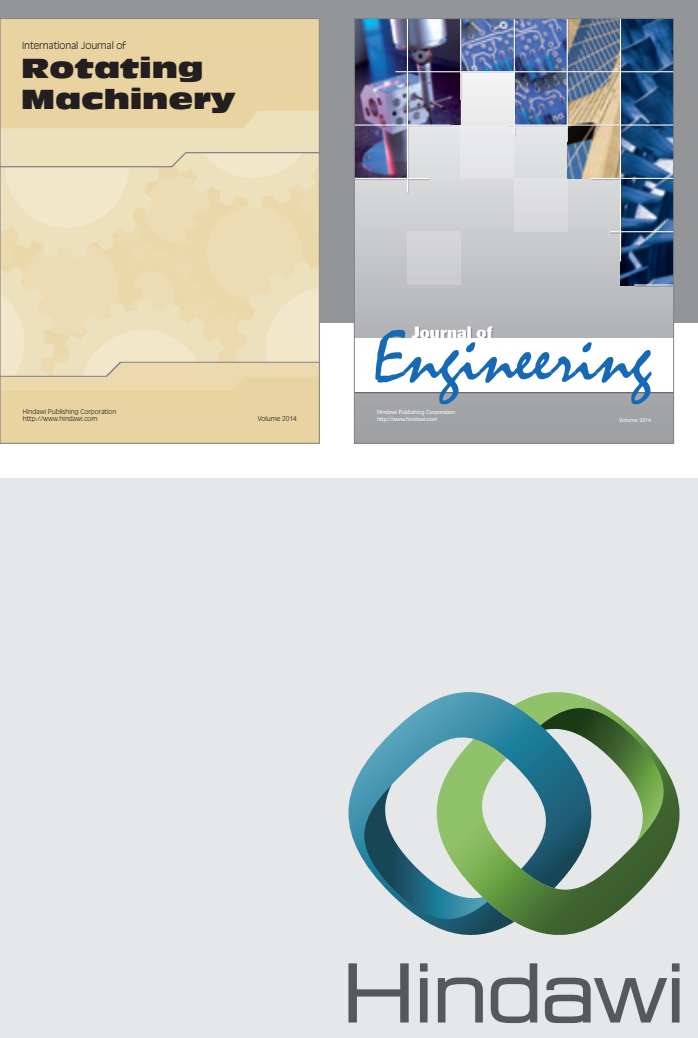

Submit your manuscripts at

http://www.hindawi.com
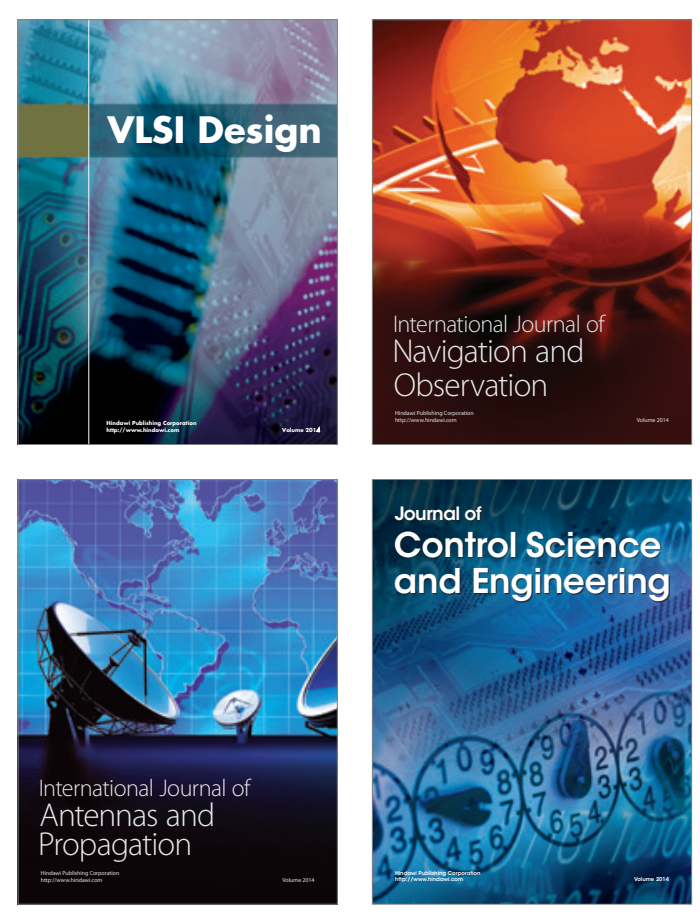
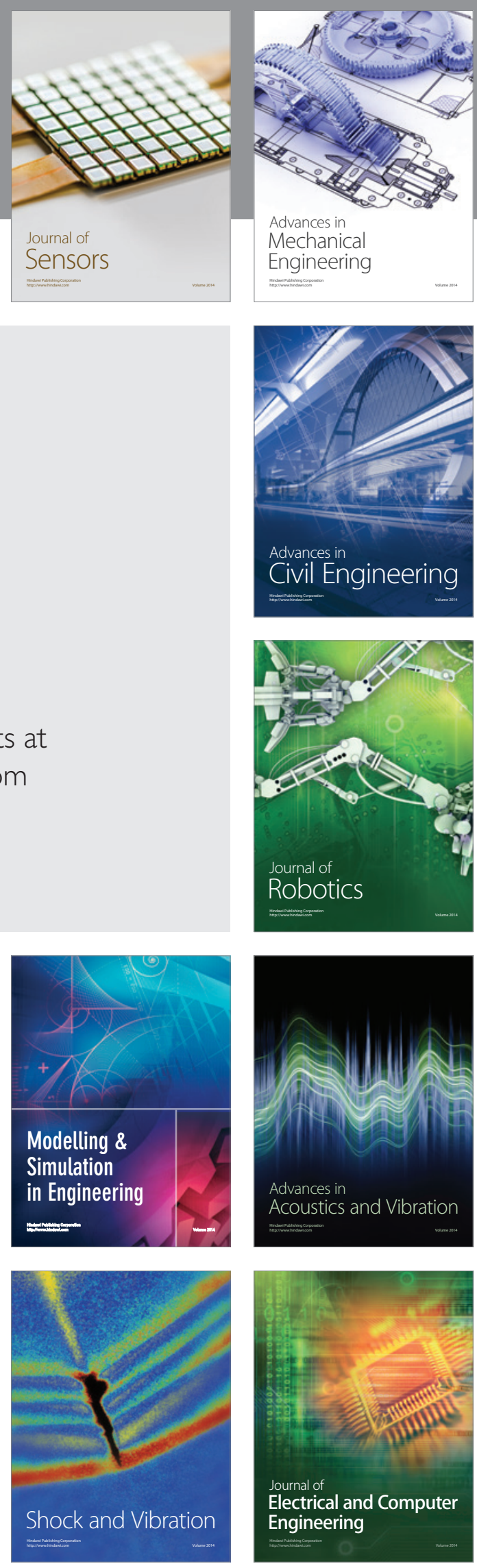